\title{
Dynamic processes and charge carriers transport in polyvinyl acetate-polyaniline composites
}

\author{
Mohammed A. Moussa' ${ }^{1}$ Mona H. Abdel Rehim² • Ahmed M. Ghoniem ${ }^{1} \cdot$ Gamal M. Turky ${ }^{1}$
}

Received: 19 December 2019 / Accepted: 12 May 2020 / Published online: 21 May 2020

(c) Springer Nature Switzerland AG 2020

\begin{abstract}
This work studies the effect of composite formation between polyvinyl acetate (PVAc) and conductive polyaniline (PAni) on its conductivity mechanism. To achieve this goal, different concentrations of PAni in mini-emulsion of PVAc are prepared. The dynamic relaxation and electric conductivity are evaluated using broadband dielectric spectroscopy technique. The relaxation rate of the local reorientations ( $\beta$-process) of ester group follows an Arrhenius relation with activation energy $39 \mathrm{~kJ} /$ mole. The coupling of the segmental dynamic ( $\alpha$-process) and terminal fluctuation ( $\beta^{\prime}$-process) occurs just above the calorimetric glass transition temperatures, $T_{g^{\prime}}$ of pure PVAc. This is reasonable since a-process that originated from a structural rearrangement became very slow below $T_{g}$ which takes the polymer toward its equilibrium state. There is also decoupling of the charge carriers transport, and the segmental motion, the concept concluded in this manuscript, sounds reasonable if one thinks about the ion motion at $T<T_{\mathrm{g}}$. This conclusion is straightforwardly deduced from the experimental data, as explained in more detail. The study also confirms that PAni electrical conductivity mechanism is conserved.
\end{abstract}

Keywords Polyvinyl acetate - Mini-emulsion · Dynamic relaxation · Conductivity · Decoupling effect

\section{Introduction}

The decoupling of the mass and charge transport, at the microscopic scale, is a challenge to be clarified and understood in polymers and polymeric composite systems. This phenomenon, shown as a transition from Vogel-Fulcher-Tammann (VFT) thermal activation's behavior of the dc conductivity at higher temperatures $\left(T_{c}>T_{g^{\prime}}\right.$ where $T_{\mathrm{g}}$ is the glass transition temperature) to Arrhenius like one at lower temperatures, was investigated in many polymeric systems [1-5]. According to Kremer et al. [6], this phenomenon was attributed to what is called tremendous demobilization of the main chain and the attached ionic groups. However, the free charge carriers (counterions) are still transported through the polymer contributing to the dc conductivity, $\sigma_{\mathrm{dc}}$. In other words, there is decoupling, at that characteristic temperature, $T_{c^{\prime}}$ between a-process originated from glassy dynamic and the charge carriers transportation contributed as dc conductivity.

Polyaniline, PAni, in its conductive form-emeraldine salt-has a great advantage due to both of its environmental and thermal stabilities. Besides, it has a tunable electrical conductivity through using different dopants and/or doping degree, so it can be used widely [7-11]. Unfortunately, it is mechanically too bad to be molded. Several researches have been done recently to improve

Electronic supplementary material The online version of this article (https://doi.org/10.1007/s42452-020-2896-8) contains supplementary material, which is available to authorized users.

$\triangle$ Mona H. Abdel Rehim, monaabdelrehim23@gmail.com | ${ }^{1}$ Microwave Physics and Dielectrics Department, National Research Centre, 33 El Behooth Street, Dokki, Giza 12622, Egypt. ${ }^{2}$ Packing and Packaging Materials Department, National Research Centre, 33 El Behooth Street, Dokki, Giza 12622, Egypt. 
its mechanical properties through doping agent and/or composite formation [12-16]. The question that appeared for composites formation is to what extent the matrix polymer's structure affect PAni electrical conductivity. To answer this query, it is necessary to investigate the effect of different polymeric matrices on the electrical conductivity mechanism of PAni. Very recently, we investigated the effect of polymethyl methacrylate [17], sulfonated polystyrene [18] and polystyrene [19], as different matrices on the conductivity of PAni. All these composites showed that the polyaniline conductivity mechanism is not affected by the polymer matrix whether in the presence or absence of the hydrogen bonding or functional groups. However, in all cases, the conductivity decreases remarkably as the matrix ratio increases as to be expected.

As dielectric relaxation phenomena are sensitive to the composite's composition, preparation method and additives, so this work aims to study the structural property relationship within a polymer composite matrix. The study also represents a follow-up for our previous investigations that involved compositing of PAni and different polymers' matrices [17-19]. To achieve this goal, PVAc is selected as a matrix. In this polymer, the ether oxygen is directly attached to the main chain instead of the carbonyl group in polymethyl methacrylate (Fig. 1). This supposed to give the carbonyl group a higher degree of freedom [20]. Therefore, this study will concentrate on investigations of polyvinyl acetate-polyaniline composites within the era of electrical dynamics and charge transport mechanisms.

The formation of interfaces due to the heterogeneous structure in this polymeric system may determine the macroscopic properties of the nano-dielectric and result in significant enhancements or detrimental effects depending on the size of the interfaces and their interactions with the polymer matrix. Dielectric spectroscopy is a powerful tool for detecting and analyzing the dynamics of polar segments that relax at the vicinity

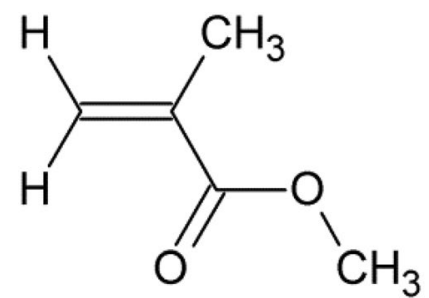

Methyl methacrylate

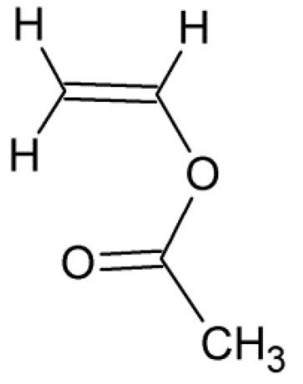

Vinyl aectate
Fig. 1 Chemical structures of methyl methacrylate and vinyl acetate monomers

SN Applied Sciences

A SPRINGER NATURE journal of nanoparticles even when the particles are at small volume fractions. This technique has been extensively described by Kremer and Schönhals and references therein [21].

\section{Experimental}

\subsection{Materials}

Aniline hydrochloride (Ani.HCl) was a product of Oxford, India. Vinyl acetate was purchased from Merck, Germany, while ammonium persulfate, hexadecane and polyvinyl alcohol were obtained from Sigma-Aldrich, Germany.

\subsection{Synthesis of polyaniline/polyvinyl acetate (PAni/ PVAc) composite}

The pure PVAc was prepared on the base of mini-emulsion polymerization procedures performed by Wang and Schork [22]. Polyvinyl acetate-polyaniline composites were prepared as described previously in some detail [23]. Briefly, a flask contained $0.2 \mathrm{~g}$ of hexadecane and $10 \mathrm{~g}$ vinyl acetate was added to a solution of polyvinyl alcohol (1.4 $\mathrm{g}$ in $24 \mathrm{ml}$ water). Then, the mixture was sonicated for $15 \mathrm{~min}$ at $100 \mathrm{w}$ in cool water/ice bath. The temperature was raised and maintained at $60 \mathrm{C}$ for about $30 \mathrm{~min}$. A solution of APS $(0.05 \mathrm{~g}$ in $0.8 \mathrm{ml}$ water) was added drowsily, allowing reaction for $2 \mathrm{~h}$. A solution of Ani. $\mathrm{HCl}$ in $10 \mathrm{ml}$ of $1 \mathrm{M} \mathrm{HCl}$ was added to the cooled mini emulsion and stirred for 30 min followed by ultrasonication for $5 \mathrm{~min}$ in cooled water bath at $100 \mathrm{~W}$. Then, $(1: 1$ molar ratios) $20 \mathrm{ml}$ APS solution in $1 \mathrm{M} \mathrm{HCl}$ was added. The reaction was maintained for $24 \mathrm{~h}$, centrifugated at $4500 \mathrm{rpm}$ for $10 \mathrm{~min}$ at $15 \mathrm{C}$ and washed three times with distilled water. Then, it is dried in oven at $60 \mathrm{C}$ overnight. The samples under investigation of different PAni/PVAC ratios are given in Table 1.
Table 1 Codes for PVAc-PAni composites

\begin{tabular}{ll}
\hline Sample & Code \\
\hline Pure polyvinyl acetate & PVAc \\
10\% PAni/PVAc & A10V \\
20\% PAni/PVAc & A20V \\
40\% PAni/PVAc & A40V \\
70\% PAni/PVAc & A70V \\
100\% PAni/PVAc & A100V \\
\hline
\end{tabular}

Weight percent here means (weight of aniline hydrochloride/weight of vinyl acetate monomers) $\times 100$ 


\subsection{Techniques}

Thermal stabilities and degradation temperatures in addition to semiquantitative analyses were performed by SDT Q600 TGA-DSC characterization instrument with heating rate $10 \mathrm{C} / \mathrm{min}$ within the temperature range $30-1000 \mathrm{C}$ in nitrogen atmosphere in an aluminum crucible.

Broadband dielectric spectroscopy (BDS) measurements were performed using a Novocontrol spectrometer (Concept 40) covering a frequency range of $10^{-1}-10^{7} \mathrm{~Hz}$ to investigate the dielectric and electrical properties of the composites at different temperatures. The frequency scans were carried out isothermally following a temperature step of $10 \mathrm{~K}$ between 223 and $393 \mathrm{~K}$. Samples for these investigations were prepared by pressing $0.5 \mathrm{~g}$ of the milled powder in stainless steel mold at $30 \mathrm{MPa}$, followed by heat pressing in Teflon sandwich at $373 \mathrm{~K}$ and $30 \mathrm{MPa}$ for film formation. Figure $\mathrm{S} 1$ shows the effect of heat molding on the ac conductivity of the investigated films. To ensure the thermal stability of the samples under heating conditions, thermal gravimetric analysis was carried out (Figure S2). The sample film was sandwiched between two brass electrodes in parallel plated capacitor cell arrangement.

The complex permittivity, $\varepsilon^{*}(v, T)=\varepsilon^{\prime}(v, T)-i \varepsilon^{\prime \prime}(v, T)$, is recorded. The relaxation modes are fitted using the model function introduced by Havriliak and Negami (HN equation) $[24,25]$. $\mathrm{HN}$ equation is a combination of the Cole-Cole and Cole-Davidson models and describes symmetric and asymmetric broadening of the dielectric function. It is read as:

$\varepsilon^{*}=\varepsilon_{\infty}+\frac{\varepsilon_{s}-\varepsilon_{\infty}}{\left(1+\left(i \omega \tau_{H N}\right)^{\beta}\right)^{\gamma}}$

where $\varepsilon_{s}$ is the relative real permittivity at zero frequency, $\varepsilon_{\infty}$ is the relative real permittivity at infinite frequency, $\tau_{H N}$ is the relaxation time, $\beta$ and $\gamma$ are the Havriliak-Negami parameters and $\omega$ is the angular frequency. It became well known that the dipolar relaxations are often hidden by dissipative losses due to Ohmic conduction.

\section{Results and discussion}

The dependence of dielectric loss, $\varepsilon^{\prime \prime}$, on temperature ranging from 223 to $393 \mathrm{~K}$ at spot point frequency $1 \mathrm{kHz}$ of pure polyvinyl acetate (PVAc) is illustrated graphically as shown in Fig. 2. This isochronal graph clarifies the existence of two dynamic relaxation processes. The primary or main process at the higher temperatures, a-process that is the cooperative segmental motion, relates to the onset of the glass transition. The low-temperature one is

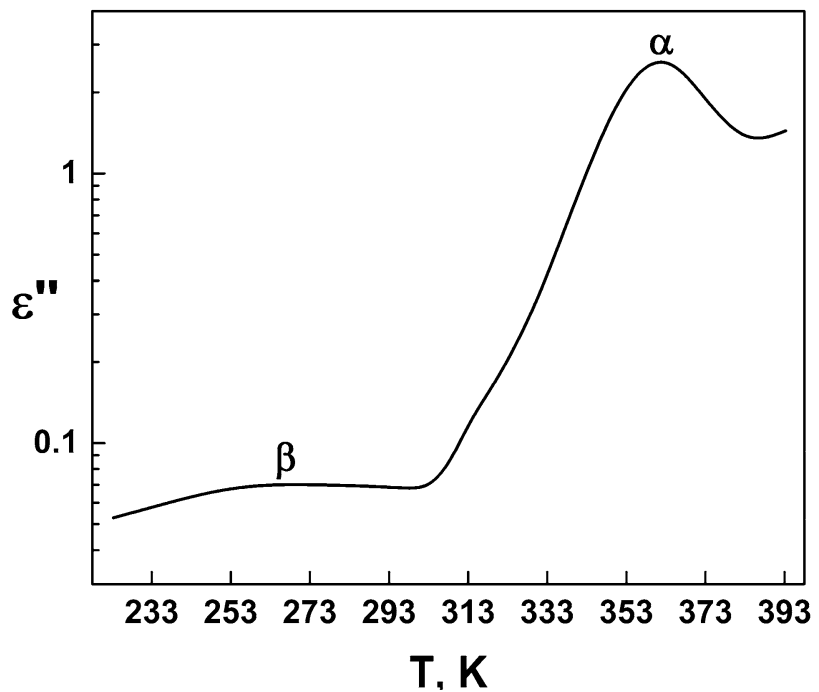

Fig. 2 Isochronal representation of dielectric loss, $\varepsilon^{\prime \prime}$, at $1 \mathrm{kHz}$ for pure polyvinyl acetate

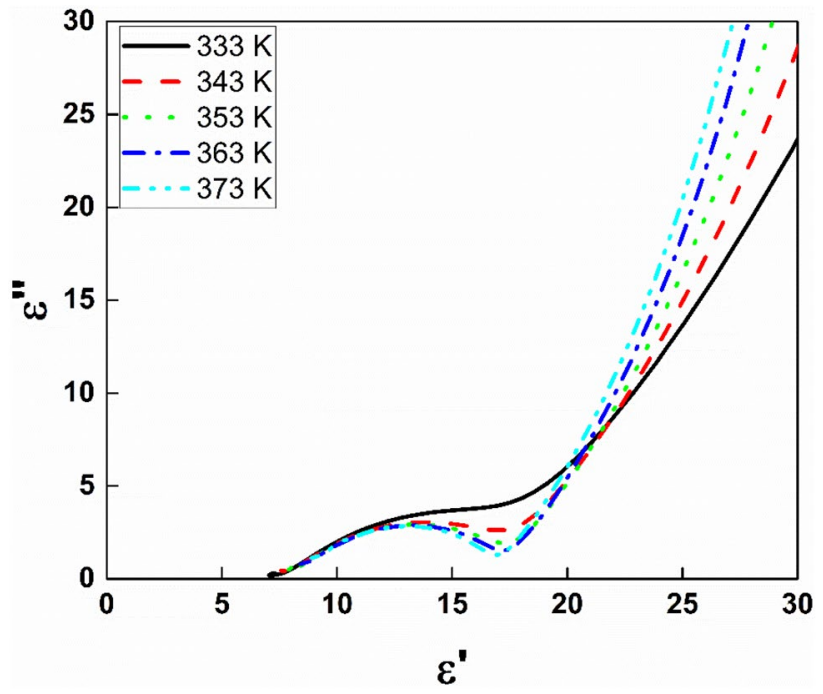

Fig. 3 Cole-Cole representation of the complex permittivity at the labeled temperatures for PVAC

a secondary dynamic called $\beta$-process that attributed to the local reorientations of the ester group. Further increase in temperature shows an overlaid that characterizes the conductivity contribution. The intensity of the a-relaxation peak is one order of magnitude higher and very sharp in comparison with that of $\beta$-relaxation peak.

Cole-Cole representation (Fig. 3) of the complex permittivity at different temperatures above the glass transition clearly shows the semicircles behavior at the high frequency for $\beta$-process and masking by conductivity at low frequency for a-process. 
The dielectric spectra of the investigated samples can be presented in terms of the complex parameters $\varepsilon^{*}, \sigma^{*}$ or $M^{*}$. The three representations (complex permittivity, electrical conductivity and modulus functions), although equivalent, i.e., directly related to each other, are often employed since they emphasize different aspects of the underlying mechanisms of charge transport and molecular dynamics. The first two forms, the complex dielectric and conductivity functions, have traditionally been used in studies of dipolar relaxations and charge transport, respectively. Maxwell's equations, which describe the interaction of electromagnetic waves with the matter, make direct reference to and prove the equivalence of these approaches. Typical dielectric spectra of PVAc as a representative example at $363 \mathrm{~K}$ measured over 8 decades in frequency are shown in Fig. 4. Three spectral regimes can be identified in fact they apparently attributable to different underlying processes. Analysis of these relaxation processes was done by fitting dielectric loss high-frequency peak with

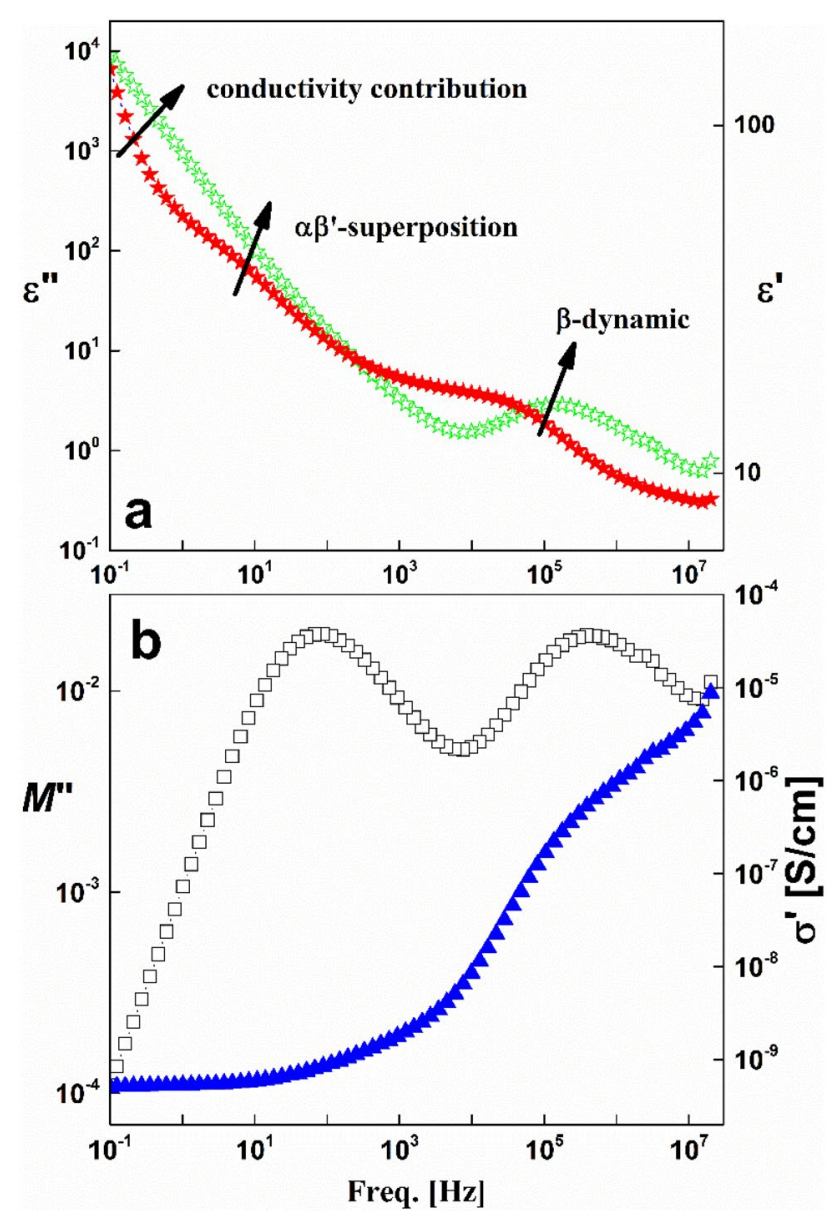

Fig. 4 The frequency dependence of a permittivity, $\varepsilon^{\prime}$ [red closed stars] and dielectric loss, $\varepsilon^{\prime \prime}$ [green open stars]; and $\mathbf{b}$ real conductivity, $\sigma^{\prime}$ (blue closed triangles) and electric loss modulus, $M^{\prime \prime}$ (black open square) for PVAc at $363 \mathrm{~K}$
Havriliak-Negami equation at temperatures below the glass transition. While at temperatures above the glass transition, it is more easy and accurate to do the fitting procedures for the imaginary modulus, $M^{\prime \prime}$, representation in order to avoid the conductivity contribution [26-28]. The complex electric modulus $M^{*}(\omega)$ is defined as the inverse of complex permittivity $\varepsilon^{*}(\omega)$ [29], indicating that

$M^{\prime \prime}=\frac{\varepsilon^{\prime \prime}}{\varepsilon^{\prime 2}+\varepsilon^{\prime \prime 2}}$

Sometimes, we need to represent the data on electric modulus $M^{*}$ representation since it is useful to describe some specific relaxation processes. Even $\varepsilon^{*}$ and $M^{*}$ are equivalent, they emphasize different aspects of the underlying mechanisms of charge transport and molecular dynamics [17]. The use of the electric modulus investigation suppresses undesirable capacitance effects due to electrode contacts and provides a clear view of dc conduction and dipole relaxation [30, 31].

The activation plots for these processes are shown in Fig. 5. At higher frequencies, secondary relaxation, whose rate follows an Arrhenius relation and has an activation energy of about $39 \mathrm{~kJ} / \mathrm{mole}$, is observed. This dynamic process was attributed to the local reorientations ( $\beta$-process) of the ester group. However, in the modulus representation, at the intermediate frequency ranges, the dielectric spectrum is governed by superimposed two dynamic processes. This superimposed process obeys Vogel-Fulcher-Tammann (VFT) activation and called a $\beta^{\prime}$ dynamic, because it is a superposition of two relaxation

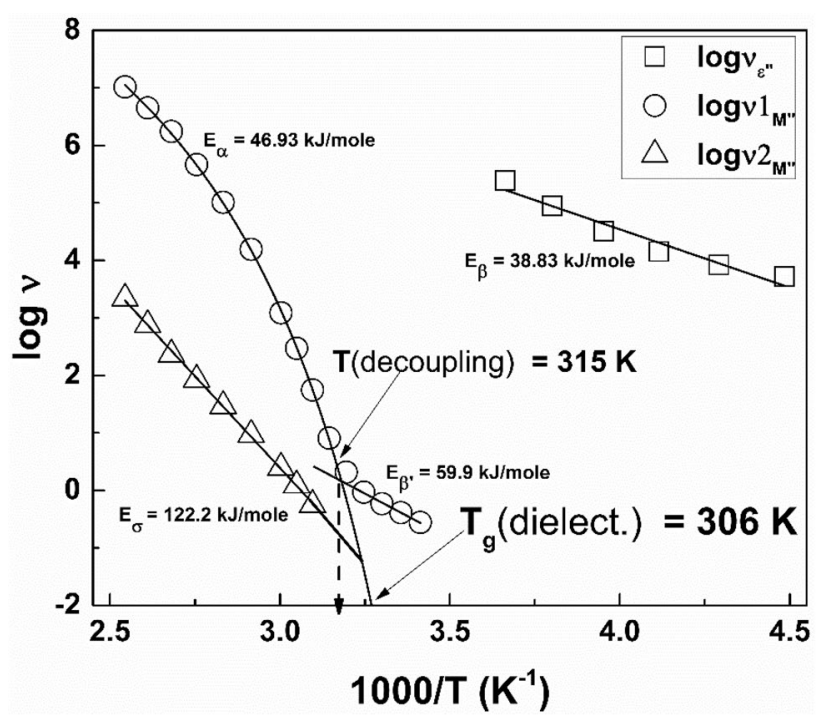

Fig. 5 Thermal activation of the $\beta$-relaxation rate from loss representation, $\alpha \beta$-relaxation rate and characteristic charge transportation process from modulus representation for PVAC 
processes, namely a-process and lower-frequency local reorientation $\beta^{\prime}$-process. The activation energy calculated using the Arrhenius equation for $\beta^{\prime}$-process is $59.9 \mathrm{~kJ} / \mathrm{mole}$, while the $\alpha \beta^{\prime}$ process has activation energy $46.9 \mathrm{~kJ} / \mathrm{mole}$ calculated in the base of VFT equation. This VFT behavior is a characteristic one for the a-process. The dielectrically determined glass transition temperature obtained by extrapolating the VFT fitting to the frequency of $0.01 \mathrm{~Hz}$ is found to be $306 \mathrm{~K}$. It is very close to the reported one using DSC technique [32, 33]. Very close to the calorimetric glass transition, $T_{\mathrm{g}}$, there is a decoupling between them that will be considered later. At lower frequencies, the conductivity contribution screened out the dynamic peak in the dielectric loss representation and characterized the transport of the charge carriers in the bulk. The process appeared at the lower frequency range attributed to the inset of hopping frequency in the conductivity representation and was found to have Arrhenius activation energy of $122.2 \mathrm{~kJ} / \mathrm{mole}$. The underlying mechanism of this contribution represents the (translational) diffusion of ions. This part of the spectra can be used to extract important molecular parameters characterizing the hopping mechanism of the charge carriers [34-38].

The decoupling between $\alpha$ - and $\beta^{\prime}$-processes appears just above the glass transition temperature. At that temperature $(315 \mathrm{~K})$, the molecular dipoles still may reorient, even in a rather rigid lattice. The a-process dynamic has the dominant peak in dielectric loss representation against both frequency and temperature in many supercooled liquid systems. Its tremendous slowing down under cooling in a narrow temperature range is accompained by the increase in viscosity. The thermal activation of the dc conductivity follows Arrhenius dependence till the glass transition temperature and then changes to follow a Vogel-Fulcher-Tammann equation. This transition was explained as a result of tremendous demobilization of the polymeric backbone including the attached ionic species, whereas the counterions are still diffusing through the polymer matrix and hence contributing to the charge transportation. It is clear that the temperature of the transition is very close to the glass transition temperature. This explained the conjecture of decoupling between charge transport and glassy dynamics, which agrees well with that found previously in some other polymeric systems $[2,39$, $1]$.

Relaxation processes in PVAc-PAni composites are greatly masked by the conductivity contribution with a more pronounced effect for the higher polyaniline ratios. The dielectric loss $\varepsilon^{\prime \prime}$, determined at a fixed temperature of $343 \mathrm{~K}$, is illustrated graphically as a function of frequency for three selected nanocomposites in Fig. 6. Therefore, we will concern our study on both the modulus and the conductivity representations.

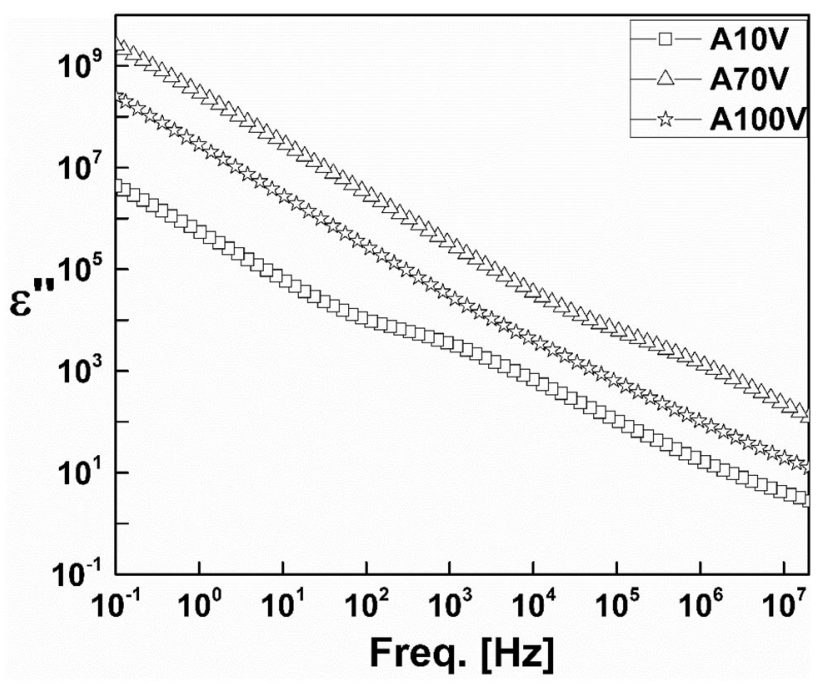

Fig. 6 Dielectric loss $\left(\varepsilon^{\prime \prime}\right)$ against frequency at $343 \mathrm{~K}$ for some PVAc-PAni composites

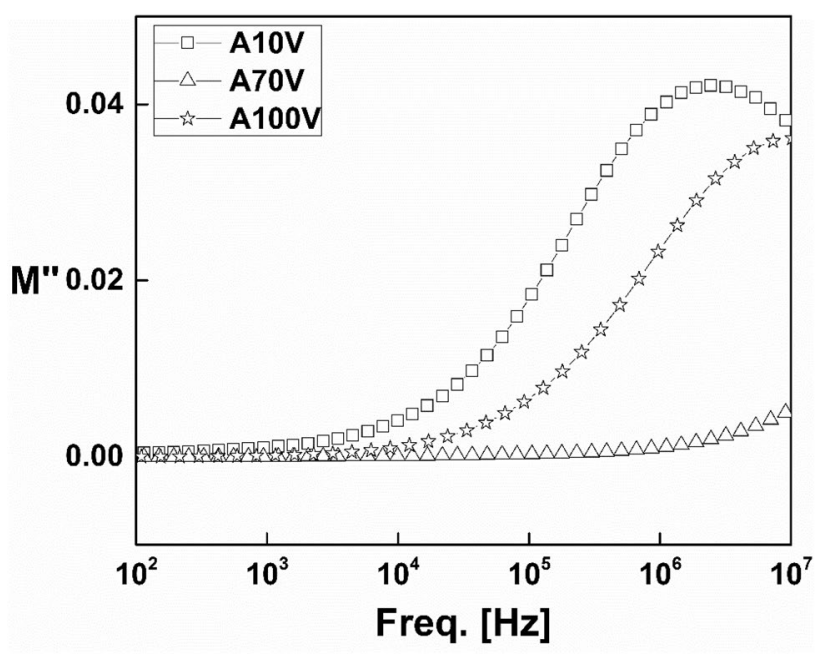

Fig. 7 Imaginary part of modulus, $M^{\prime \prime}$, against frequency at $293 \mathrm{~K}$ for some PVAc-PAni composites

Figure 7 clarifies the variation of the imaginary part of modulus with frequency for A10V, A70V and A100V at $293 \mathrm{~K}$. In the high frequency range, asymmetric relaxation process is detected and shifted to a higher frequency for higher polyaniline concentration, but more accurately for the higher PAni-PVAc composites' conductivity. Therefore, this process is out of the measuring frequency window for A70V. This process cannot be considered as MWS one (usually called interfacial polarization) due to its low relaxation strength and the temperature slowing-down effect.

In order to estimate the origin of this process, the isothermal data at a series of temperatures were fitted using the Havriliak-Negami equation. The fitting results are 
shown in Fig. 8. The relaxation rate increases linearly with temperature up to near the glass transition temperature $\left(T_{g}=306 \mathrm{~K}\right)$. The $\beta$-process describes the fluctuation of the ester group of the polyvinyl acetate $\left(\mathrm{CH}_{3} \mathrm{COO}\right)$ with some accompanying methylene groups. That electric loss modulus representation shows that this process became faster as the free volume increases that give more degrees of freedom. We assume in the lower-temperature range that the rigid polyaniline chain serves as a spacer between PVAc chains, and hence the available free volume increases for the relaxation group associated with $\beta$-process. The increase in PAni ratio supposed to decrease the steric hindrance; therefore, the process becomes faster and has lower activation energy as shown in Table 2. The expected water traces may build some hydrogen bonding with the carbonyl groups and hence gives a chance for PAni chain to interact with the PVAc carbonyl groups, and so greater activation energy would be postulated for that process. In addition, this increase in the activation energy at higher temperatures could be assigned to PAni structural changes that occur at this range of temperatures (323-333 K).

Figure 9 shows the variation of ac conductivity with frequency at $323 \mathrm{~K}$ for the blank polyvinyl acetate and its PAni composites. It shows a dc plateau in the lower frequency range followed by power-law increasing region after a certain characteristic frequency. A strong bend in the power-law region, especially for the low PAni ratios, is shown. It is attributed, in such complicated system, to the Maxwell-Wagner-Siller (MWS) polarization. This polarization originates from the charge carriers accumulated at the interfaces between the two polymers. That bend is assumed to be due to the MWS process, and not the segmental motion (a-dynamic) because it is more

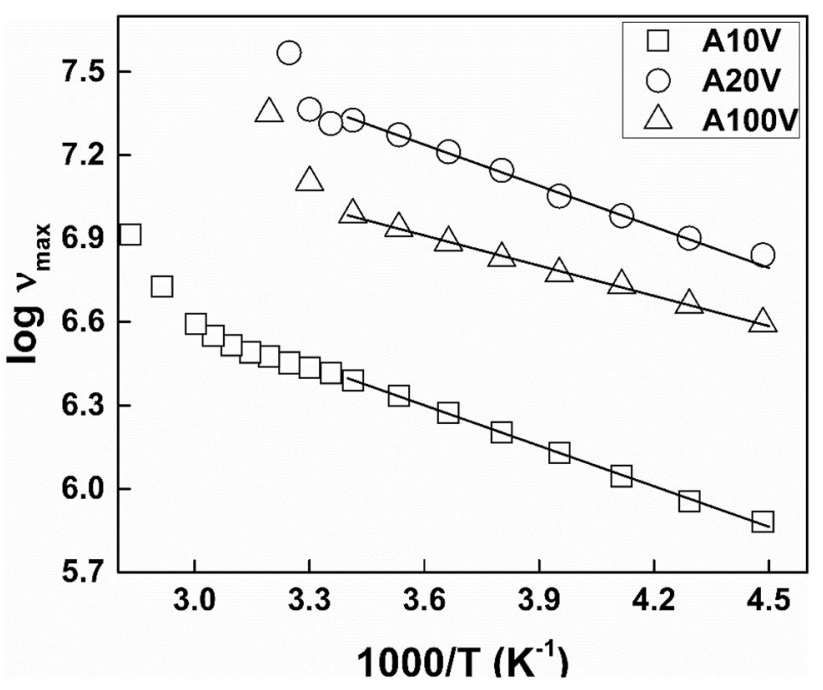

Fig. 8 Activation plot of the imaginary part of modulus, $M^{\prime \prime}$, relaxation peak for some PVAc-PAni composites
Table 2 Activation energies for $\beta$-process $\left(E_{\beta}\right)$ and dc conductivity $\left(E_{\sigma_{d c}}\right)$ in the lower-temperature range for PVAc-PAni composites

\begin{tabular}{lll}
\hline Sample & $E_{\beta}(\mathrm{kJ} /$ mole $)$ & $E_{\sigma_{\mathrm{dc}}}(\mathrm{kJ} / \mathrm{mole})$ \\
\hline A10V & 9.3 & 20.1 \\
A20V & 9.4 & 14.2 \\
A40V & 9.0 & 13.6 \\
A70V & - & 13.4 \\
A100V & 6.9 & 10.4 \\
\hline
\end{tabular}

pronounced for the lower PAni ratio since the interfacial area will vanish for the higher polyaniline loadings. The conductivity increases five orders of magnitude by PAni addition (A10V), then follows the same sequence observed in the previous works for composites of PMMA [17] and PS [19] and can be attributed to the effect of viscosity of the reaction medium on the ordering of polyaniline chains. Dyre fit of the isothermal conductivity at a series of temperatures will result in the activation plot of dc conductivity as shown in Fig. 10. The activation plot in the lower temperature range follows an Arrhenius relation. This activation energies decrease with increasing polyaniline concentration (Table 2 ) since the increase in the charge carrier density by raising polyaniline ratio (Figure S3) will occupy energy levels closer to the conduction band on the base of band theory.

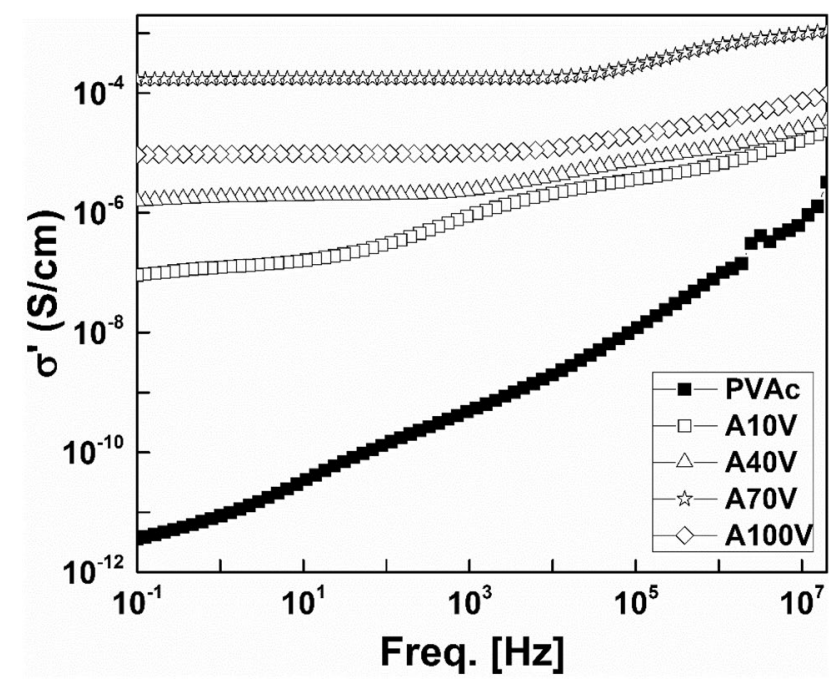

Fig. 9 Real part of conductivity $\left(\sigma^{\prime}\right)$ against frequency at $323 \mathrm{~K}$ for the blank polyvinyl acetate (PVAc), in addition to its polyaniline composites 


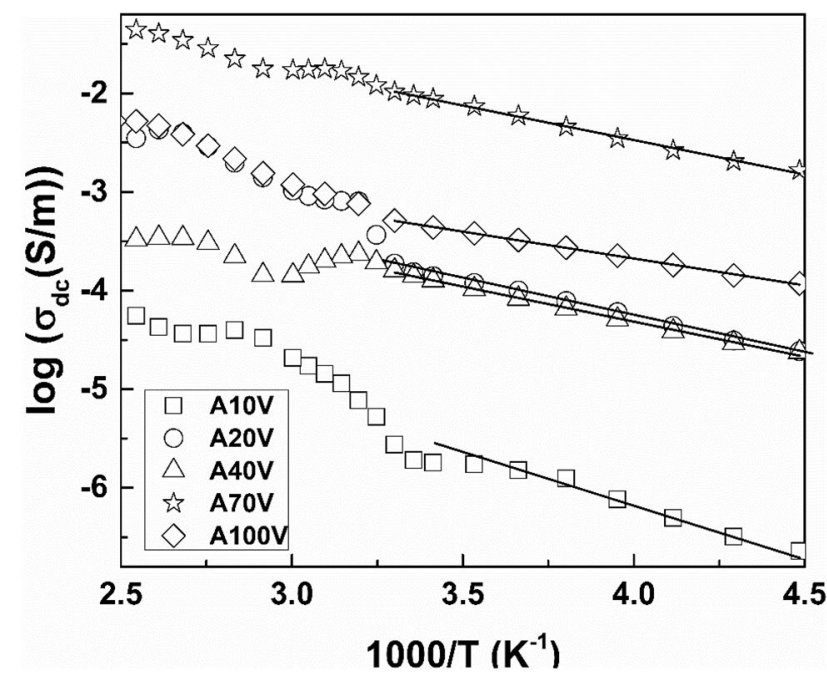

Fig. 10 Activation plot of the dc conductivity for PVAc-PAni composites. The solid lines are the Arrhenius fit in the lower temperature range

\section{Conclusion}

Polyvinyl acetate-polyaniline composites are prepared with the mini-emulsion method. Decoupling of $\beta^{\prime}$-process from a-process occurs at the glass transition temperature. Polyaniline rigid chain could serve as spacers between polyvinyl acetate chains and increase the free volume for $\beta^{\prime}$-process's reorientation. Polyaniline electrical conductivity mechanism is conserved and is not affected by the presence of polyvinyl acetate.

Acknowledgements Financial support for this work by the National Research Centre research (Projects No. 11050105) is greatly acknowledged.

\section{Compliance with ethical standards}

Conflict of interest On behalf of all authors, the corresponding author states that there is no conflict of interest.

\section{References}

1. Wojnarowska Z, Knapik J, Jacquemin J, Berdzinski S, Strehmel V, Sangoro JR, Paluch M (2015) Effect of Pressure on Decoupling of Ionic Conductivity from Segmental Dynamics in Polymerized Ionic Liquids. Macromolecules 48:8660-8666

2. Choi UH, Ye Y, de la Cruz DS, Liu W, Winey KI, Elabd YA, Runt J, Colby RH (2014) Dielectric and Viscoelastic Responses of Imidazolium-Based lonomers with Different Counterions and Side Chain Lengths. Macromolecules 47:777-790

3. Fan $F$, Wang $W$, Holt AP, Feng $H$, Uhrig $D$, Lu $X$, Hong $T$, Wang Y, Kang N-G, Mays J, Sokolov AP (2016) Effect of Molecular
Weight on the Ion Transport Mechanism in Polymerized Ionic Liquids. Macromolecules 49:4557-4570

4. Zhao M (2014) Thermal Analysis and Dielectric Spectral Characteristics of Poly(ionic Liquids): Towards exploration of their utility in capacitive electrochemical devices, Ph.D. Thesis, Rochester Institute of Technology, Rochester, New York, USA.

5. Wojnarowska Z, Knapik J, Diaz M, Ortiz A, Ortiz I, Paluch M (2014) Conductivity mechanism in polymerized imidazolium-based protic ionic liquid [ $\left.\mathrm{HSO}_{3}-\mathrm{BVIm}\right][\mathrm{OTf}]$ : dielectric relaxation studies. Macromolecules 47:4056-4065

6. Frenzel F, Borchert P, Anton A, Strehmel V, Kremer F (2019) Charge transport and glassy dynamics in polymeric ionic liquids as reflected by their inter- and intramolecular interactions. Soft Matter 15:1605-1618

7. Liao G, Li Q, Xu Z (2019) The chemical modification of polyaniline with enhanced properties: a review. Prog Org Coat 126:35-43

8. Huang W, Xiaoa Y, Huang Z, Tsui G, Yeung K, Tang C, Liu Q (2020) Super-hydrophobic polyaniline- $\mathrm{TiO}_{2}$ hierarchical nanocomposite as anticorrosion coating. Mater Lett 258:126822

9. Zhang F, Xiong P, Guo X, Zhang J, Yang W, Wu W, Liu H, Wang $G$ (2019) A nitrogen, sulphur dual-doped hierarchical porous carbon with interconnected conductive polyaniline coating for high-performance sodium-selenium batteries. Energy Storage Mater 19:251-260

10. Liu P, Yan J, Guang Z, Huang Y, Li X, Huang W (2019) Recent advancements of polyaniline-based nanocomposites for supercapacitors. J Power Sources 424:108-130

11. Duan J, Duan Y, Zhao Y, Wang Y, Tang Q, He B (2018) Bifunctional polyaniline electrode tailored hybridized solar cells for energy harvesting from sun and rain. J Energy Chem 27:742-747

12. Monicka K, Polpaya IC, Varughese S (2018) Sulphosuccinic acid as a novel dopant for solution processable polyaniline. Mater Chem Phys 217:357-364

13. Oliveira R, Bizeto M, Camilo F (2018) Production of self-supported conductive films based on cellulose, polyaniline and silver nanoparticles. Carbohydr Polym 199:84-91

14. Saini P, Jalan R, Dhawan SK (2008) Synthesis and characterization of processable polyaniline doped with novel dopant NaSIPA. J App Poly Sci 108(3):1437-1446

15. Wu GY, Zhang L, Yu D, Wu M, Hni H (2018) Enhanced electrical conductivity and competent mechanical properties of polyaniline/polyacrylate (PANI/PA) composites for antistatic finishing prepared at the aid of polymeric stabilizer. Prog Org Coat 125:99-108

16. Araujo JR, Lopes ES, deCastro RK, Senna CA, Robertis E, Neves RS, Fragneaud B, Nykänen A, Kuznetsov A, Archanjo BS, De Paoli MA (2018) Chapter 8: characterization of polyaniline-based blends, composites, and nanocomposites. Elsevier, Amsterdam, Netherlands, pp 209-233

17. Moussa MA, Ghoneim AM, Abdel Rehim MH, Khairy ShA, Soliman MA, Turky GM (2017) Relaxation dynamic and electrical mobility for poly(methyl methacrylate)-polyaniline composites. J Appl Polym Sci 134(42):45415

18. Moussa MA, Abdel Rehim MH, Khairy ShA, Soliman MA, Aghoneim AM, Turky GM (2015) Electrical investigations of polyaniline/sulfonated polystyrene composites using broadband dielectric spectroscopy. Synth Met 209:34-40

19. Moussa MA, Abdel Rehim MH, Ghoneim AM, Khairy ShA, Solimanc MA, Turky GM (2019) Dielectric investigations and charge transport in PS-PAni composites with ionic and nonionic surfactants. J Phys Chem Solids 133:163-170

20. Zhang Y, Fan H, Wang Y, Zuo B, Zhang W, Wang S, Wang X (2015) Influence of the linkage type between the polymer backbone and side groups on the surface segregation of methyl groups during film formation. Soft Matter 11:9168-9178 
21. Kremer F, Schönhals A (2003) Broadband dielectric spectroscopy. Springer, Berlin

22. Wang S, F.J. Schork FJ, (1994) Miniemulsion polymerization of vinyl acetate with nonionic surfactant. J Appl Polym Sci 54(13):2157-2164

23. Moussa MA (2017) Preparation of plastic electrodes based on conductive hybrid polymers. Ph.D. Thesis, Physics Department, Cairo University, Giza, Egypt.

24. Havriliak S, Negami S (1966) A complex plane analysis of a-dispersions in some polymer systems. J Poly Sci Part C 14:99-117

25. Havriliak S, Negami S (1967) A complex plane representation of dielectric and mechanical relaxation processes in some polymers. Polymer 8:161-210

26. Dacrory S, Abou-Yousef H, Kamel S, Turky G (2019) Devolvement of biodegradable semiconducting foam based on micro-fibrillated cellulose/Cu-NPs. Int J Biol Macromol 132:351-359

27. Darwish WM, Abd El-Ghaffar M, Turky G (2014) Synthesis and electric modulus formalism of novel metal-phthalocyanine bridged polymers. J Inorg Organomet Polym Mater 24:858-864

28. Omara S, Turky G, Ghoneim A, Thünemann A, Abdel Rehim M, Schönhals A (2017) Hyperbranched poly (amidoamine)/kaolinite nanocomposites: structure and charge carrier dynamics. Polymer 121:64-74

29. Griffin P, Agapov A, Kisliuk A, Sun X, Dai S, Novikov V, Sokolov A (2011) Decoupling charge transport from the structural dynamics in room temperature ionic liquids. J Chem Phys 135:114509

30. Papathanassiou AN, Mykhailiv O, Echegoyen L, Sakellis I, Plonska-Brzezinska ME (2016) Electric properties of carbon nanoonion/polyaniline composites: a combined electric modulus and ac conductivity study. J. Phys. D: Appl. Phys. 48:285305

31. Kolonelou E, Papathanassiou AN, Sakellis E (2019) Evidence of local softening in glassy poly(vinyl alcohol)/poly(vinyl pyrrolidone) $(1 / 1, w / w)$ nano-graphene platelets composites. Mat. Chem, Phys. 223:140-144
32. Svoboda R, Pustková P, Málek J (2008) Structural relaxation of polyvinyl acetate (PVAc). Polymer 49:3176

33. Drioli E, Giorno L (2015) Encyclopedia of membranes, Johannes Carolus Jansen, glass transition temperature. Springer, Berlin

34. Gainaru C, Stacy E, Bocharova V, Gobet M, Holt A, Saito T, Greenbaum S, Sokolov A (2016) Mechanism of conductivity relaxation in liquid and polymeric electrolytes: direct link between conductivity and diffusivity. J Phys Chem B 120:11074-11083

35. Frenzel F, Binder W, Sangoro J, Kremer F (2016) Ch 5. Glassy dynamics and charge transport in polymeric ionic liquids. In: Paluch M (ed) Dielectric Properties of Ionic Liquids. Springer, Berlin

36. lacob C, Matsumoto A, Brennan M, Liu H, Paddison S, Urakawa O, Inoue T, SangoroJ RJ (2017) Polymerized ionic liquids: correlation of ionic conductivity with nanoscale morphology and counterion volume. ACS Macro Lett 6:941-946

37. Turky G, Sangoro J, Abdel Rehim M, Kremer F (2010) Secondary relaxations and electrical conductivity in hyperbranched polyester amides. Polym Sci Part: B Polym Phys 48:1651-1657

38. Sangoro J, Turky G, Abdel Rehim M, Jacob C, Naumov S, Ghoneim A, Kärger J, Kremer F (2009) Charge transport and dipolar relaxations in hyperbranched polyamide amines. Macromolecules 42:1648-1651

39. Fan F, Wang Y, Hong T, Heres M, Saito T, Sokolov A (2015) Ion conduction in polymerized ionic liquids with different pendant groups. Macromolecules 48:4461-4470

Publisher's Note Springer Nature remains neutral with regard to jurisdictional claims in published maps and institutional affiliations. 